\title{
sensors
}

ISSN 1424-8220

(C) 2002 by MDPI

http://www.mdpi.net/sensors

\section{Asymptotic Regional Boundary Observer in Distributed Parameter Systems via Sensors Structures}

\section{Raheam Al-Saphory}

Systems Theory Laboratory, University of Perpignan, 52, avenue de villeneuve, 66860 Perpignan, France. E-mail : saphory@univ-perp.fr

Received: 6 March 2002 / Accepted: 4 April 2002 / Published: 26 April 2002

\begin{abstract}
The purpose of this paper is to study the concept of asymptotic regional observer in connection with the characterizations of sensors. We give various results related to different types of measurements, of domains and boundary conditions. Furthermore, we show that the measurements structures allow the existence of regional observer and we give a sufficient condition for such observer. We also show that, there exists a dynamical system for the considered system is not observer in the usual sense, but it may be regional boundary observer.
\end{abstract}

Key words: Sensors, $\Gamma$-detectability, $\Gamma$-observers, Diffusion system.

\section{Introduction}

The observer theory was introduced by Luenberger in [1] and is generalized to infinite dimensional control systems described by semi-group operators in [2]. The analysis of distributed parameter systems has received much attention in the literatures $[3,4,5]$. The study of this notion, through the structure of the sensors and the actuators, was developed by El Jai and Pritchard $[6,7]$.

The notion of regional analysis was extended by El Jai and Zerrik et al. [8, 9]. This study motivated by certain concrete-real problems, in thermic, mechanic, environment $[10,11]$. If a system defined on a domain $\Omega$ is represented as in the (Fig. 1), then we are interested in the regional state on $\omega$ or $\Gamma$ of the domain $\Omega$.

The concept of asymptotic regional analysis was introduced recently by Al-Saphory and El Jai in $[12,13,14]$, consists in studying the behavior of systems not in all the domain $\Omega$ but only on particular regions $\omega$ or $\Gamma$ of the domain. In this paper, we are only interested in the asymptotic regional boundary state on a region $\Gamma$ of the boundary $\partial \Omega$. The purpose of this paper is to give some results related to the 
link between the $\Gamma$-observer and the number of sensors, their locations, the geometrical domains and boundary conditions.

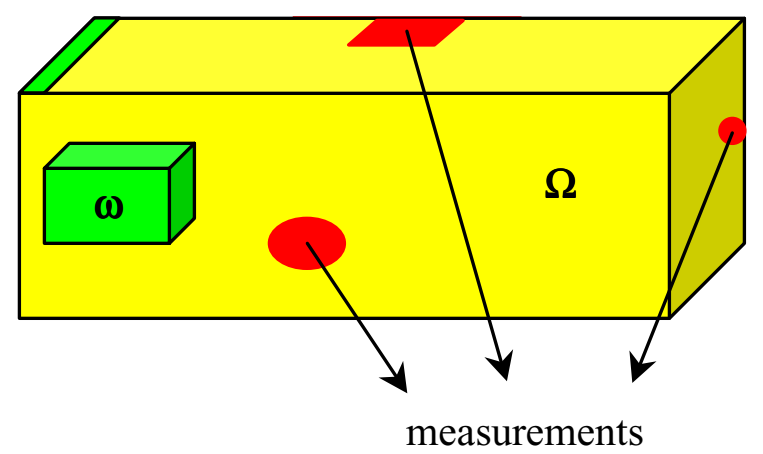

Figure 1. Domain $\Omega$, regions $\omega, \Gamma$ and locations of measurements.

The paper is organized as follows. Section 2 concerns the formulation problem and preliminaries. Section 3, devotes to the introduction of $\Gamma$-detectability problem. In section 4 , we give some definitions and characterizations concerns $\Gamma$-observer and strategic sensors. We show that there exists a counter-example of the case $\Gamma$-observer which is not observer, in the whole domaine. In the last section, we illustrate applications with various situations of sensor locations and we characterize such a regional boundary observer to a diffusion system.

\section{Problem Statement}

Let $\Omega$ be a regular, bounded and open set of $R^{n}$, with smooth boundary $\partial \Omega$ and $\Gamma$ be a nonempty given subregion of $\partial \Omega$, with positive measurement. We denote $Q=\Omega \times] 0, \infty[$ and $\Theta=\partial \Omega \times] 0, \infty[$. We consider the system described by the following parabolic equation

$$
\left\{\begin{array}{llrl}
\frac{\partial z}{\partial t}(\xi, t) & =A z(\xi, t)+B u(t) & & \bar{\Omega} \\
z(\xi, t) & =z_{0}(\xi) & \Theta \\
\frac{\partial z}{\partial v}(\eta, t) & =0 &
\end{array}\right.
$$

with the output function

$$
y(., t)=C z(., t)
$$

and we assume that $Z, U, O$ be separable Hilbert spaces where $Z$ is the state space, $U$ the control space and $O$ the observation space. Usually we consider $Z=H^{1}(\Omega), U=L^{2}\left(0, \infty, R^{p}\right)$ and $O=L^{2}(0, \infty$, $R^{q}$ ) where $p$ and $q$ hold for the number of actuators and sensors. $A$ is a second order linear differential operator which generates a strongly continuous semi-group $\left(S_{A}(t)\right)_{t \geq 0}$ on $Z$ and is selfadjoint with compact resolvent. The operators $B \in L\left(R^{p}, Z\right)$ and $C \in L\left(Z, R^{\mathrm{q}}\right)$ depend on the structure of actuators and sensors [7]. Under the given assumptions, the system (2.1) has an unique solution given by 


$$
z(., t)=S_{A}(t) z_{0}(.)+\int_{0}^{t} S_{A}(t-\tau) B u(\tau) d \tau
$$

The measurements can be obtained by the use of zone, pointwise or lines sensors which may be located in $\Omega$ (or $\partial \Omega$ ) [7].

- Let us recall that a sensor by any couple $(D, f)$ where $D$ denote closed subsets of $\bar{\Omega}$, which is spatial support of sensor and $f \in L^{2}(D)$ defines the spatial distribution of measurement on $D$. According to the choice of the parameters $D$ and $f$, we have various types of sensors. A sensors may be a zone types when $D \subset \Omega$. The output function (2.2) can be written in the form

$$
y(t)=C z(., t)=\int_{D} z(\xi, t) f(\xi) d \xi
$$

A sensor may also be a point when $D=\{b\}$ and $f=\delta(.-b)$ where $\delta$ is the Dirac mass concentrated in $b$. Then, the output function (2.2) may be given by the form

$$
y(t)=C z(., t)=\int_{\Omega} z(\xi, t) \delta_{b}(\xi-b) d \xi
$$

In the case of boundary zone sensor, we consider $D=\Gamma$ with $\Gamma \subset \partial \Omega$ and $f \in L^{2}(\Gamma)$. The output function (2.2) can then be written in the form

$$
y(t)=C z(., t)=\int_{\Gamma} z(\eta, t) f(\eta) d \eta
$$

The operator $C$ is unbounded and some precautions must be taken in $[7,15]$.

- $\quad$ The operator $K$ defined by

$$
\begin{aligned}
K: H^{1}(\Omega) & \rightarrow L^{2}\left(0, \infty, R^{\mathrm{q}}\right) \\
z & \rightarrow C S_{A}(t) z
\end{aligned}
$$

and in the case of internal zone sensors is, linear and bounded with an adjoint

$$
\begin{aligned}
K^{*}: H^{1}(\Omega) & \rightarrow L\left(0, \infty, R^{q}\right) \\
y^{*} & \rightarrow \int_{0}^{t} S_{A}^{*}(\tau) C^{*} y^{*} d \tau
\end{aligned}
$$

- The trace operator of order zero

$$
\gamma_{0}: H^{1}(\Omega) \rightarrow H^{1 / 2}(\partial \Omega)
$$

is linear, surjective and continuous with adjoint denoted by $\gamma_{0}{ }^{*}$

- Consider a subdomain $\Gamma$ of $\partial \Omega$ and let $\chi_{\Gamma}$ be the function defined by

$$
\begin{aligned}
\chi_{\Gamma}: H^{1 / 2}(\partial \Omega) & \rightarrow H^{1 / 2}(\Gamma) \\
z & \rightarrow \chi_{\Gamma} z=z
\end{aligned}
$$

where $z_{\left.\right|_{\Gamma}}$ is the restriction of the state $z$ to $\Gamma$. We denote by $\chi_{\Gamma}^{*}$ the adjoint of $\chi_{\Gamma}$.

- Let $\chi_{\omega}$ be the function defined by

$$
\begin{aligned}
\chi_{\omega}: H^{1}(\Omega) & \rightarrow H^{1}(\omega) \\
z & \rightarrow \chi_{\omega} z=z_{\mid \omega}
\end{aligned}
$$

where $z_{\mid \Gamma}$ is the restriction of the state $z$ to $\omega$. 
- The operator $T_{\Gamma}: H^{1}(\Omega) \rightarrow H^{1 / 2}(\Gamma)$ is given by

$T_{\Gamma}=\chi_{\Gamma} \gamma_{0} T$

Recalling some definitions concern the notion of $\Gamma$-observability as in $[9,16]$ :

- The autonomous system associated to (2.1)-(2.2) is said to be exactly (respectively weakly)

$\Gamma$-observable if :

$\operatorname{Im} \chi_{\Gamma} \gamma_{0} K^{*}=H^{1 / 2}(\Gamma) \quad$ (respectively $\left.\overline{\operatorname{Im} \chi_{\Gamma} \gamma_{0} K^{*}}=H^{1 / 2}(\Gamma)\right)$

- The suite $\left(D_{i}, f_{i}\right)_{1 \leq i \leq q}$ of sensors is said to be $\Gamma$-strategic if the system (2.1) together with the output function (2.2) is weakly $\Gamma$-observable. For the dual results concerning the actuators structures [17].

\section{Regional boundary detectability}

The main reason for introducing $\Gamma$-detectability is, the possibility to construct an $\Gamma$-estimator for the current state of the original system. This concept is extended by Al-Saphory

and El Jai in [13]. For this objective we recall some definitions concern this concept :

- The system (2.1) is said to be $\partial \Omega$-stable, if the operator $A$ generates a semi-group which is stable on the space $H^{1 / 2}(\partial \Omega)$. It is easy to see that the system (2.1) is $\partial \Omega$-stable, if and only if, for some positive constants $M$ and $\alpha$, we have

$$
\left\|\gamma_{0} S_{A}(.)\right\|_{H^{1 / 2}(\partial \Omega)} \leq M e^{-\alpha t}, \forall t \geq 0
$$

If $\left(S_{A}(t)\right)_{t \geq 0}$ is stable semi-group $H^{1 / 2}(\partial \Omega)$, then for all $z_{0} \in H^{1}(\Omega)$, the solution of the associated autonomous system satisfies

$$
\lim _{t \rightarrow \infty}\left\|\gamma_{0} z(., t)\right\|_{H^{1 / 2}(\partial \Omega)}=\lim _{t \rightarrow \infty}\left\|\gamma_{0} S_{A} z_{0}(.)\right\|_{H^{1 / 2}(\partial \Omega)}=0
$$

- The system (2.1) together with the output (2.2) is said to be $\partial \Omega$-detectable, if there exists an operator $H_{\partial \Omega}: R^{q} \rightarrow H^{1 / 2}(\partial \Omega)$ such that $\left(A-H_{\partial \Omega} C\right)$ generates a strongly continuous semi-group $\left(S_{H_{\partial \Omega}}(t)\right)_{t \geq 0}$ which is stable on $H^{1 / 2}(\partial \Omega)$.

- If a system is $\partial \Omega$-detectable, then it is possible to construct an asymptotic $\partial \Omega$-observer for the original system. If we consider the system

$$
\begin{cases}\frac{\partial x}{\partial t}(\xi, t)=A x(\xi, t)+B u(t)+H_{\partial \Omega}(y(., t)-C y(\xi, t)) & \bar{\Omega} \\ x(\xi, t)=x_{0}(\xi) & \Theta \\ \frac{\partial x}{\partial v}(\eta, t)=0 & \end{cases}
$$

then $x(\xi, t)$ estimates asymptotically the state $z(\xi, t)$ because the error $e(\xi, t)=z(\xi, t)-x(\xi, t)$ satisfies $\frac{\partial e}{\partial t}(\xi, t)=\left(A-H_{\partial \Omega} C\right) e(\xi, t)$ with $e(\xi, t)=z(\xi, t)-x(\xi, t)$. Then, if the system is $\partial \Omega$-detectable, it is possible to choose $H_{\partial \Omega}$ which realizes $\lim _{t \rightarrow \infty}\|e(., t)\|_{H^{1 / 2}(\partial \Omega)}=0$. 
Remark 3.1. In this paper, we only need the relation (3.1) to be true on a given subregion $\Gamma$ of the region $\partial \Omega$

$$
\lim _{t \rightarrow \infty}\left\|\chi_{\Gamma} \gamma_{0} z(., t)\right\|_{H^{1 / 2}(\Gamma)}=\lim _{t \rightarrow \infty}\left\|\chi_{\Gamma} \gamma_{0} S_{A} z_{0}(.)\right\|_{H^{1 / 2}(\Gamma)}=0
$$

we may refer to this as $\Gamma$-stability.

- The system (2.1) is said to be $\Gamma$-stable, if the operator $A$ generates a semi-group which is stable on $H^{1 / 2}(\Gamma)$.

- The system (2.1)-(2.2) is said to be $\Gamma$-detectable if there exists an operator $H_{\Gamma}: R^{q} \rightarrow H^{1 / 2}(\Gamma)$ such that $\left(A-H_{\Gamma} C\right)$ generates a strongly continuous semi-group $\left(S_{H_{\Gamma}}(t)\right)_{t \geq 0}$ which is stable on $H^{1 / 2}(\Gamma)$.

For more detail [18]. However, one can easily have the following results :

Corollary 3.2. If the system (2.1) together with output function (2.2) is exactly $\Gamma$-observable, then it is $\Gamma$-detectable.

This result leads to:

$\exists \gamma>0$ such that $\left\|\chi_{\Gamma} \gamma_{0} S_{A} z_{0}(.)\right\|_{H^{1 / 2}(\Gamma)}=\left\|C S_{A} z_{0}(.)\right\|_{L^{2}(0, \infty, O)}, \forall z_{0} \in H^{1 / 2}(\Gamma)$

Thus, the notion of $\Gamma$-detectability is a weaker property than the exact $\Gamma$-observability as in (Ref. [16].

\section{Asymptotic $\Gamma$-observer and strategic sensors}

In this section, we propose an approach which allows to determinate a regional asymptotic estimator of $z(\xi$, $t)$ on $\Gamma$, based on the internal asymptotic $\Gamma$-observer. This method need some definitions concern regional observer as in [11].

\subsection{Definitions and characterizations}

- Consider the system (2.1) - (2.2) together with the dynamical system

$$
\left\{\begin{array}{lr}
\frac{\partial x}{\partial t}(\xi, t)=F_{\omega} x(\xi, t)+G_{\omega} u(t)+H_{\omega} y(t) & \bar{\Omega} \\
x(\xi, t)=x_{0}(\xi) & \Theta \\
\frac{\partial x}{\partial v}(\eta, t)=0 &
\end{array}\right.
$$

where $F_{\omega}$ generates a strongly continuous semi-group $\left(S_{F_{\omega}}(t)\right)_{t \geq 0}$ which is stable on $X=H^{1}(\omega)$, i.e. :

$$
\exists M_{F_{\omega}}, \alpha_{F_{\omega}}>0 \text { such that }\left\|\chi_{\omega} S_{F_{\omega}}(.)\right\|_{H^{1}(\omega)} \leq M_{F_{\omega}} e^{-\alpha_{F_{\omega}} t}
$$

and $G_{\omega} \in L\left(R^{p}, H^{1}(\omega)\right)$ and $H_{\omega} \in L\left(R^{q}, H^{1}(\omega)\right)$. The system (4.1) defines an asymptotic $\omega$ estimator for $\chi_{\omega} T(\xi, t)$ where $z(\xi, t)$ is a solution of the system (2.1)-(2.2) if 
$\lim _{t \rightarrow \infty}\left\|x(., t)-\chi_{\omega} T(., t)\right\|_{H^{1}(\omega)}=0$ and $\chi_{\omega} T$ maps $D(A)$ into $D\left(F_{\omega}\right)$ where $x(\xi, t)$ is the solution of the system (4.1).

- The system (4.1) specifies an $\omega$-observer for the system (2.1)-(2.2) if the following conditions hold:

1. There exists $M_{\omega} \in L\left(R^{q}, L^{2}(\omega)\right)$ and $N_{\omega} \in L\left(L^{2}(\omega)\right)$ such that $M_{\omega} C+N_{\omega} \chi_{\omega} T=I_{\omega}$.

2. $\chi_{\omega} T A+F_{\omega} \chi_{\omega} T=G_{\omega} C$ and $H_{\omega}=\chi_{\omega} T B$.

3. The system (4.1) defines an asymptotic $\omega$-estimator for $\chi_{\omega} T(\xi, t)$.

- The system (4.1) is said to be an identity $\omega$-observer for the system (2.1)-(2.2) if and $Z=X$.

- The system (4.1) is said to be a reduced-order $\omega$-observer for the system (2.1)-(2.2) if $Z=O \oplus X$.

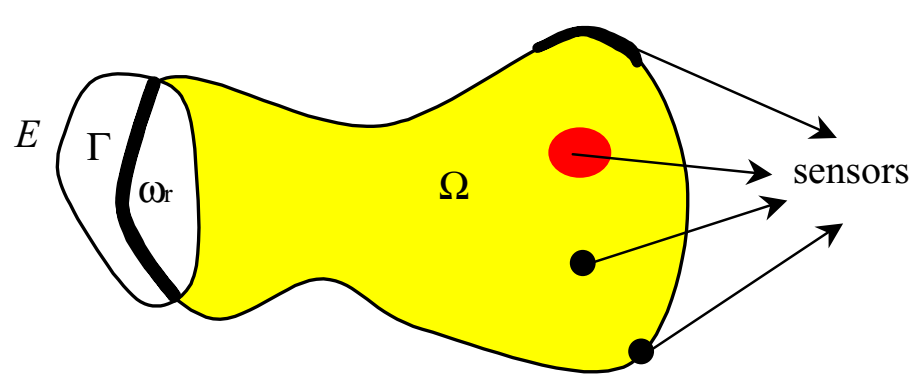

Figure 2: The considered domain $\Omega$ and the subregion $\omega_{r}$.

- The boundary regional observer in $\Gamma$ may be seen as internal regional observer in $\omega$ if we consider the following transformations. Let $\mathfrak{R}$ be the continuous linear extension operator [19] $\Re: H^{1 / 2}(\partial \Omega) \rightarrow H^{1}(\Omega)$ such that

$$
\gamma_{0} \Re h(\xi, t)=h(\xi, t), \quad \forall h \in H^{1 / 2}(\partial \Omega)
$$

- Let $r>0$ is an arbitrary and sufficiently small real and let the sets

$$
E=\bigcup_{z \in \Gamma} B(z, r) \text { and } \omega_{r}=E \cap \Omega
$$

where $B(z, r)$ is the ball of radius $r$ centered in $z(\xi, t)$ and where $\Gamma$ is a part of $\bar{\omega}_{r}$ (Fig. 2).

Proposition 4.1. If the system (2.1)-(2.2) is exactly (respectively weakly) $\bar{\omega}_{r}$-observable, then it is exactly (respectively weakly) $\Gamma$-observable (see [9]).

From this result, we can deduce the following proposition :

Proposition 4.2. The dynamical system (4.1) is Luenberger $\bar{\omega}_{r}$-observer for the system (2.1) - (2.2), then it is Luenberger $\Gamma$-observer.

Proof: Let $x(\xi, t) \in H^{1 / 2}(\Gamma)$ and $\bar{x}(\xi, t)$ its extension to $H^{1 / 2}(\partial \Omega)$ using (4.3) and trace theorem, there exists $\Re \bar{x}(\xi, t) \in H^{1}(\Omega)$ with a bounded support such that 


$$
\gamma_{0} \Re \bar{x}(\xi, t)=\bar{x}(\xi, t)
$$

Since the system (4.1) is regional observer on $\bar{\omega}_{r}$ so we can deduce that :

The system (4.1) is regional observer on $\omega_{r}$, there exists a dynamical system with $x(\xi, t) \in X$ such that

$$
\chi_{\omega} T z(\xi, t)=\chi_{\omega} \Re \bar{x}(\xi, t)
$$

then we have

$$
\chi_{\Gamma}\left(\gamma_{0} \chi_{\omega}^{*} \chi_{\omega} T z\right)(\xi, t)=x(\xi, t)
$$

The equations (2.2) and (4.5) allow

$$
\left[\begin{array}{l}
y \\
x
\end{array}\right](\xi, t)=\left[\begin{array}{c}
C \\
\chi_{\Gamma}\left(\gamma_{0} \chi_{\omega}^{*} \chi_{\omega} T\right)
\end{array}\right] z(\xi, t)
$$

and there exist two linear bounded operators $\bar{R}$ and $S$ satisfy the relation

$$
\bar{R} C+\chi_{\mathrm{r}}\left(\gamma_{0} \chi_{\omega}^{*} \chi_{\omega} T\right)=I_{\mathrm{r}}
$$

There exists an operator $F_{\bar{\omega}_{r}}$ is regionally stable on $\bar{\omega}_{r}$, then it is regionally stable on $\Gamma[18]$. Finally the system (4.1) is a regional boundary observer on $\Gamma$.

\subsection{Sufficient condition for $\Gamma$-observer}

As in (Refs. [7,18]), we develop a characterized result that links the $\Gamma$-observer and strategic sensors and we give a sufficient condition for $\Gamma$-observer. For that purpose, we assume that there exists a complete set of eigenfunctions $\varphi_{n}$ of $A$ in $H^{1}(\Omega)$, associated to the eigenvalues $\lambda_{n}$ with a multiplicity $s_{n}$ and suppose that the functions $\psi_{n}$ defined by $\psi_{n}=\chi_{\Gamma} \gamma_{0} \varphi_{n}(\xi)$, is a complete set in $H^{1 / 2}(\Gamma)$. If the system (2.1) has $J$ unstable modes, then we have the following theorem.

Theorem 4.3. Suppose that there are q zone sensors $\left(D_{i}, f_{i}\right)_{1 \leq i \leq q}$ and the spectrum of A contains $J$ eigenvalues with non-negative real parts. If the following conditions are satisfied :

1. $q>s$

2. $\operatorname{rank} G_{n}=r_{n}, \forall n, n=1, \ldots, J$ with

$$
G_{n}=\left(G_{n}\right)_{i j}= \begin{cases}\left\langle\psi_{n_{j}}(.), f(.)\right\rangle_{L^{2}(D)} & \text { zone sensor case } \\ \psi_{n_{j}}(b) & \text { pointwise sensor case } \\ \left\langle\psi_{n_{j}}(.), f(.)\right\rangle_{L^{2}(\Gamma)} & \text { boundary zone sensor case }\end{cases}
$$

where $\sup s_{n}=s<\infty$ and $j=1, \ldots, s_{n}$. Then the dynamical system 


$$
\left\{\begin{array}{lll}
\frac{\partial x}{\partial t}(\xi, t) & =A x(\xi, t)+B u(t)-H_{\Gamma} C(x(\xi, t)-z(., t)) & Q \\
x(\xi, t) & =x_{0}(\xi) & \bar{\Omega} \\
\frac{\partial x}{\partial v}(\eta, t)=0 & \Theta
\end{array}\right.
$$

is $\Gamma$-observer for the system $(2.1)-(2.2)$, i.e. $\lim _{t \rightarrow \infty}\left\|x(., t)-T_{\Gamma} z(., t)\right\|_{H^{1 / 2}(\Gamma)}=0$.

Proof: The proof is limited to the case of zone sensors in the following stapes :

Step 1. Under the assumptions of section 2, the system (2.1) can be decomposed by the projections $P$ and $I-P$ on two parts, unstable and stable. The state vector may be given by where $z_{1}(\xi, t)$ is the state component of the unstable part of the system (2.1), may be written in the form

$$
\left\{\begin{array}{llr}
\frac{\partial z_{1}}{\partial t}(\xi, t) & =A z_{1}(\xi, t)+B u(t) & \\
z_{1}(\xi, t) & =z_{0_{1}}(\xi) & \bar{\Omega} \\
\frac{\partial z_{1}}{\partial v}(\eta, t) & =0 & \Theta
\end{array}\right.
$$

and $z_{2}(\xi, t)$ is the component state of the stable part of the system (2.1) given by

$$
\begin{cases}\frac{\partial z_{2}}{\partial t}(\xi, t)=A z_{21}(\xi, t)+B u(t) & \\ z_{2}(\xi, t)=z_{0_{2}}(\xi) & \bar{\Omega} \\ \frac{\partial z_{2}}{\partial v}(\eta, t)=0 & \Theta\end{cases}
$$

The operator $A_{1}$ is represented by a matrix of order $\left(\sum_{n=1}^{J} s_{n}, \sum_{n=1}^{J} s_{n}\right)$ given

$$
A_{1}=\operatorname{diag}\left[\lambda_{1}, \ldots, \lambda_{1}, \ldots, \lambda_{2}, \ldots, \lambda_{2}, \ldots, \lambda_{J}, \ldots, \lambda_{J}\right] \text { and } P B=\left[G_{1}^{t r}, G_{2}^{t r}, \ldots, G_{J}^{t r}\right]
$$

Step 2. The condition (2) of this theorem, allows that the suite $\left(D_{i}, f_{i}\right)_{1 \leq i \leq q}$ of sensors is $\Gamma$ strategic, the subsystem (4.7) is weakly $\Gamma$-observable [16] and since it is finite dimensional, then it is exactly $\Gamma$-observable. Therefore it is $\Gamma$-detectable, and hence there exists an operator $H_{\Gamma}^{1}$ such that $\left(A_{1}-H_{\Gamma}^{1} c\right)$ which is satisfied the following :

$$
\exists M_{\Gamma}^{1}, \alpha_{\Gamma}^{1}>0 \text { such that }\left\|e^{\left(\mathrm{A}_{1}-H_{\Gamma}^{1}\right) t}\right\|_{H^{1 / 2}(\Gamma)} \leq M_{\Gamma}^{1} e^{-\alpha_{\Gamma}^{1} t}
$$

and then we have

$$
\left\|z_{1}(., t)\right\|_{H^{1 / 2}(\Gamma)} \leq M_{\Gamma}^{1} e^{-\alpha_{\Gamma}^{1} t}\left\|P z_{0_{1}}(.)\right\|_{H^{1 / 2}(\Gamma)}
$$

Since the semi-group generated by the operator $A_{2}$ is stable on $H^{1 / 2}(\Gamma)$, then there exist $\bar{M}_{\Gamma}, \bar{\alpha}_{\Gamma}>0$ such that 


$$
\left\|z_{2}(., t)\right\|_{H^{1 / 2}(\Gamma)} \leq \bar{M}_{\Gamma} e^{-\bar{\alpha}_{\Gamma} t}\left\|(I-P) z_{0_{2}}(.)\right\|_{H^{1 / 2}(\Gamma)}+\int_{0}^{t} \bar{M}_{\Gamma} e^{-\bar{\alpha}_{\Gamma}(t-\tau)}\left\|(I-P) z_{0_{2}}(.)\right\|_{H^{1 / 2}(\Gamma)}\|u(\tau)\| d \tau
$$

and therefore $\|z(\xi, t)\|_{H^{1 / 2}(\Gamma)} \rightarrow 0$ when $t \rightarrow \infty$. Finally, the system (2.1) - (2.2) is $\Gamma$-detectable.

Step 3. Let $e(\xi, t)=z(\xi, t)-x(\xi, t)$ where $x(\xi, t)$ is the solution of the system (4.6). Deriving the above equation and using the equations (2.1) and (4.6), we obtain

$$
\begin{aligned}
\frac{\partial e}{\partial t}(\xi, t) & =\frac{\partial z}{\partial t}(\xi, t)-\frac{\partial x}{\partial t}(\xi, t) \\
& =A z(\xi, t)-A x(\xi, t)-H_{\Gamma} \quad(z(., t)-x(\xi, t)) \\
& =\left(A-H_{\Gamma} C\right) e(\xi, t)
\end{aligned}
$$

Since the system (2.1)-(2.2) is $\Gamma$-detectable, there exists an operator $H_{\Gamma} \in L\left(R^{q}, H^{1 / 2}(\Gamma)\right)$, such that the operator $\left(A-H_{\Gamma} C\right)$, generates a stable, strongly continuous semi-group $\left(S_{H_{\Gamma}}(t)\right)_{t \geq 0}$ on the space $H^{1 / 2}(\Gamma)$ which is satisfied the following relations :

$$
\exists M_{\Gamma}, \alpha_{\omega}>0 \text { such that }\left\|\chi_{\Gamma} \gamma_{0} S_{H_{\Gamma}}(.)\right\|_{H^{1 / 2}(\Gamma)} \leq M_{\Gamma} e^{-\alpha_{\Gamma} t}
$$

Finally, we have

$$
\|e(., t)\|_{H^{1 / 2}(\Gamma)} \leq\left\|\chi_{\Gamma} \gamma_{0} S_{H_{\Gamma}}(.)\right\|_{H^{1 / 2}(\Gamma)}\left\|e_{0}(.)\right\| \leq M_{\Gamma} e^{-\alpha_{\Gamma} t}\left\|e_{0}(.)\right\|
$$

with $e_{0}(\xi, t)=z_{0}(\xi, t)-x_{0}(\xi, t)$ and therefore

$$
\lim _{t \rightarrow \infty}\|e(., t)\|_{H^{1 / 2}(\Gamma)}=0
$$

Thus, the dynamical system (4.6) a $\Gamma$-observer for the system $(2.1)-(2.2)$.

Remark 4.4. From theorem 4.3., we can deduce the following statements :

1. A dynamical system which is an $\partial \Omega$-observer is $\Gamma$-observer.

2. If a system is $\Gamma$-observer, then it is $\Gamma_{1}$-observer in every subset $\Gamma_{1}$ of $\Gamma$, but the converse is not true. This may be proven in the following example:

Example 4.5. Consider a two-dimensional system described by the diffusion equation

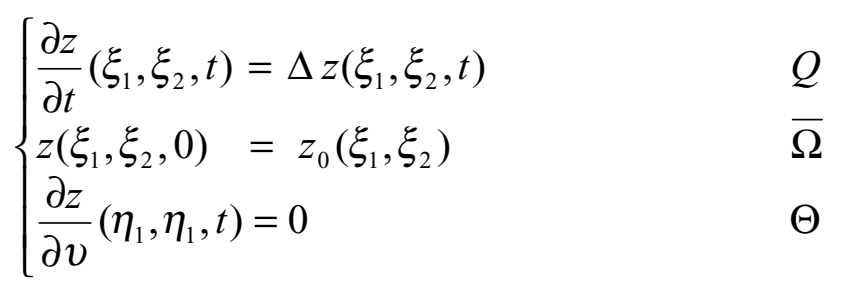

where $\Omega=] 0,1[\times] 0,1\left[\right.$. The operator $A=\Delta$ generates a strongly continuous semi-group $\left(S_{A}(t)\right)_{t \geq 0}$ on the Hilbert space $H^{1}(\Omega)$ given by

$$
S_{A}(t) z=\sum_{n, m=0}^{\infty} e^{\lambda_{n m}} t<z, \varphi_{n m}>_{H^{1}(\Omega)} \varphi_{n m}
$$


where $\lambda_{n m}=-\left(n^{2}+m^{2}\right) \pi^{2}, \varphi_{n m}\left(\xi_{1}, \xi_{2}\right)=2 a_{n m} \cos \left(n \pi \xi_{1}\right) \cos \left(m \pi \xi_{2}\right)$ and $2 a_{n m}=\left(1-\lambda_{n m}\right)^{-1 / 2}$.

Consider the dynamical system

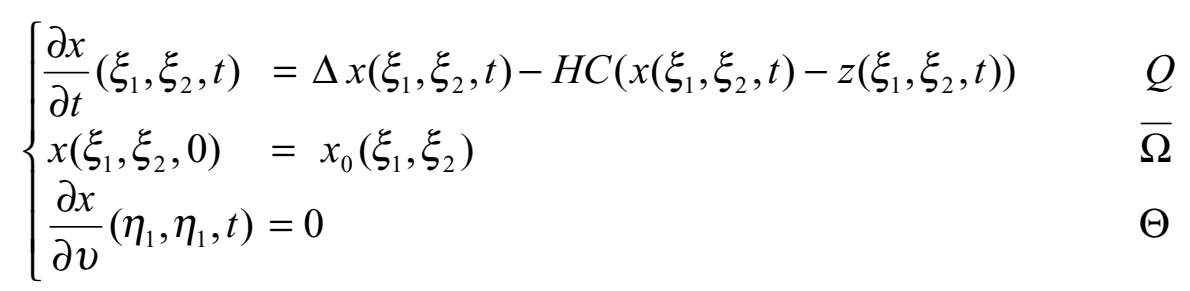

where $H \in L\left(R^{q}, Z\right), Z$ is a Hilbert space and $C: H^{1}(\bar{\Omega}) \rightarrow R^{q}$ is a linear operator. Consider the boundary sensor $\left(\Gamma_{0}, f\right)$ defined by $\left.\Gamma_{0}=\{0\} \times\right] 0,1\left[\right.$ and $f\left(\eta_{1}, \eta_{2}\right)=\cos \pi \eta_{2}$. Thus, the output function can be written by

$$
y(t)=\int_{\Gamma_{0}} z\left(\eta_{1}, \eta_{2}, t\right) f\left(\eta_{1}, \eta_{2}\right) d \eta_{1} d \eta_{2}
$$

If the state $z_{0}$ is defined in $\Omega$ by $z_{0}\left(\xi_{1}, \xi_{2}\right)=\cos \left(\pi \xi_{1}\right) \cos \left(2 \pi \xi_{2}\right)$, then the system (4.9) - (4.11) is not weakly observable in $\Omega$, i.e. the sensor $\left(\Gamma_{0}, f\right)$ is not strategic and therefore the system (4.9) - (4.11) is not detectable in $\Omega$. Thus, the dynamical system (4.10) is not observer for the system (4.9) - (4.11) (see [6]). Here, we consider the region $\Gamma=] 0,1[\times\{0\} \subset \partial \Omega$ (Fig. 3) and the dynamical system

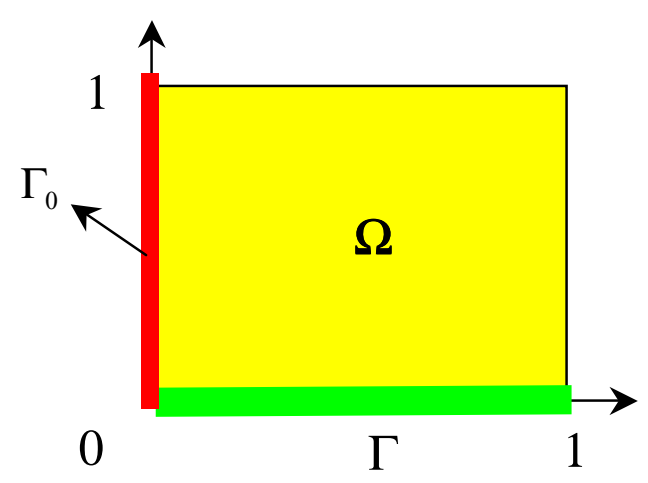

Figure 3. Domain $\Omega$, region $\Gamma$ and location $\Gamma_{0}$ of zone sensor.

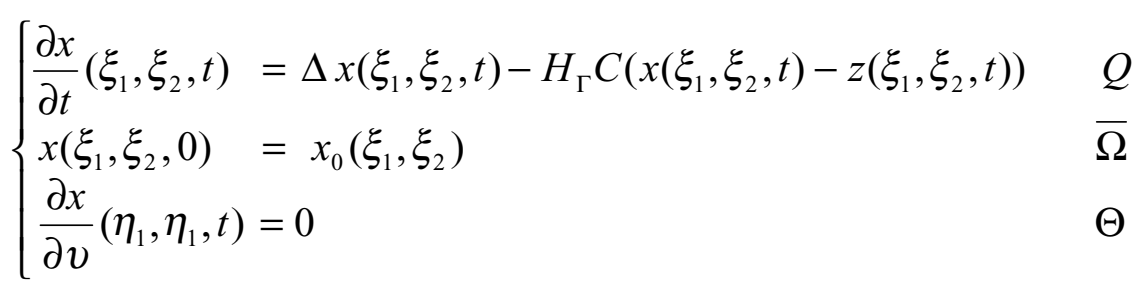

where $H \in L\left(R^{q}, H^{1 / 2}(\Gamma)\right)$. In this case, the system (4.9)-(4.11) is weakly observable in $\Gamma$ and the sensor $\left(\Gamma_{0}, f\right)$ is $\Gamma$-strategic [9]. Thus, the system (4.9)-(4.11) is $\Gamma$-detectable [13]. Finally the dynamical system (4.9) is $\Gamma$-observer for the system (4.9) - (4.11) [18]. 


\section{Application to sensors structures}

In this section, we consider the distributed diffusion systems defined on $\Omega=] 0, a_{1}[\times] 0, a_{2}[$. We explore various results related to different types of measurements, domains and boundary conditions.

\subsection{Case of a zone sensor}

We study the following cases :

\subsubsection{Rectangular domain}

In this case, the system (2.1) - (2.2) is given by

$$
\left\{\begin{array}{lll}
\frac{\partial z}{\partial t}\left(\xi_{1}, \xi_{2}, t\right) & =\Delta z\left(\xi_{1}, \xi_{2}, t\right)+B u(t) & \\
z\left(\xi_{1}, \xi_{2}, 0\right) & =z_{0}\left(\xi_{1}, \xi_{2}\right) & \bar{\Omega} \\
\frac{\partial z}{\partial v}\left(\eta_{1}, \eta_{2}, t\right) & =0 & \Theta \\
y(t) & =\int_{\Omega} z\left(\eta_{1}, \eta_{2}\right) f\left(\eta_{1}, \eta_{2}\right) d \eta_{1} d \eta_{2} & \Sigma
\end{array}\right.
$$

where $\Sigma=\bar{\Omega} \times] 0, \infty[, \mathrm{D} \subset \Omega$ is the location of zone sensor and above system represent the heatconduction problem (see Ref. [20]). Let $\left.\Gamma=\left\{a_{1}\right\} \times\right] 0, a_{2}[$ be a region of on $] 0, a_{1}[\times] 0, a_{2}[$ The eigenfunctions and the eigenvalues are given by

$$
\psi_{n m}\left(\xi_{1}, \xi_{2}\right)=\frac{2}{\sqrt{a_{1} a_{2}}} \cos \left(n \pi \frac{\xi_{1}}{a_{1}}\right) \cos \left(m \pi \frac{\xi_{2}}{a_{2}}\right)
$$

and

$$
\lambda_{n m}=\left(\frac{n^{2}}{a_{1}^{2}}+\frac{m^{2}}{a_{2}^{2}}\right) \pi^{2}
$$

If $a_{1}^{2} / a_{2}^{2} \notin \mathrm{Q}$, then the multiplicity of $s_{\mathrm{nm}}=1$ and one sensor can be guaranteed $\Gamma$-strategic sensor (see [21]). The dynamical system (4.6) may be given by



Let the measurement support is rectangular with $D=\left[\xi_{1}-l_{1}, \xi_{1}+l_{1}\right] \times\left[\xi_{2}-l_{2}, \xi_{2}+l_{2}\right] \in \Omega$.

If $f_{1}$ is symmetric about $\xi_{1}=\xi_{0_{1}}$ and $f_{2}$ is symmetric with respect to $\xi_{2}=\xi_{0_{2}}$, then we have the following result :

Corollary 5.1. The dynamical system (5.4) is $\Gamma$-observer for the system (5.1) if $n \xi_{0_{1}} / a_{1}$ and $m \xi_{0_{2}} / a_{2} \notin N$ for every $n, m=1, \ldots, J$. 
In the case where $\Gamma \subset \partial \Omega$ and $f \subset L^{2}(\Gamma)$, the sensor $(D, f)$ may be located on the boundary in $\Gamma_{0}=\left[\eta_{0_{1}}-l, \eta_{0_{1}}+l\right] \times\left\{a_{2}\right\}$ then we have :
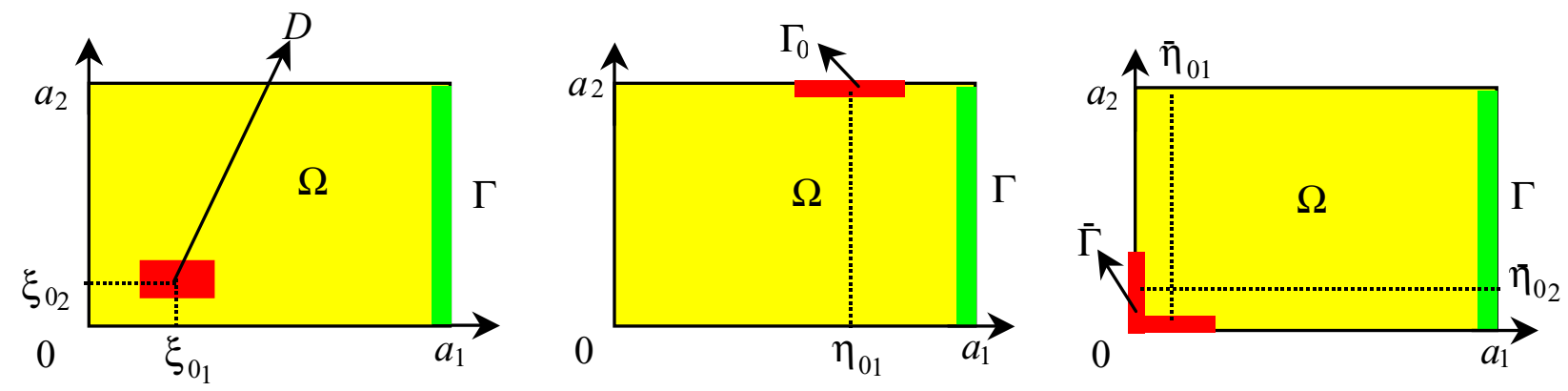

Figure 4. Domain $\Omega$, region $\Gamma$ and locations $D, \Gamma_{0}, \bar{\Gamma}$ of zone sensor.

\section{Corollary 5.1.}

1. One side case : Suppose that the sensor $(D, f)$ is located on $\Gamma_{0}=\left[\eta_{0_{1}}-l, \eta_{0_{1}}+l\right] \times\left\{a_{2}\right\} \subset \partial \Omega$ and $f$ is symmetric with respect to $\eta_{1}=\eta_{0_{1}}$, then the dynamical system (5.4) is $\Gamma$ - observer for the system (5.1) if $n \eta_{0_{1}} / a_{1} \notin N$ for every $n, m=1, \ldots, J$.

2. Two side case : Suppose that the sensor $(D, f)$ is located on $\bar{\Gamma}=\left[0, \bar{\eta}_{0_{1}}+l_{1}\right] \times\{0\} \cup\{0\}$ $\times\left[0, \bar{\eta}_{0_{2}}+l_{2}\right] \times \subset \partial \Omega$ and $f_{\left.\right|_{\Gamma_{1}}}$ is symmetric with respect to $\eta_{1}=\bar{\eta}_{0_{1}}$ and the function $f_{\left.\right|_{\Gamma_{2}}}$ is symmetric with respect to $\eta_{2}=\bar{\eta}_{0_{2}}$, then the dynamical system (5.4) is $\Gamma$-observer for the system (5.1) if $n \bar{\eta}_{0_{1}} / a_{1}$ and $m \bar{\eta}_{0_{2}} / a_{2} \notin N$ for every $n, m=1, \ldots, J$.

\subsubsection{Disk domain}

The system (5.1) may be given by the following form

$$
\left\{\begin{array}{lll}
\frac{\partial z}{\partial t}(r, \theta, t) & =\Delta z(r, \theta, t)+B u(t) & \\
z(r, \theta, 0) & =z_{0}(r, \theta) & \bar{\Omega} \\
\frac{\partial z}{\partial v}(a, \theta, 0) & =0 & \Theta \\
y_{i}(t) & =\int_{\bar{\Omega}} z\left(r_{i}, \theta_{i}, t\right) f\left(r_{i}, \theta_{i}\right) d r_{i} d \theta_{i} & \Sigma
\end{array}\right.
$$

where $0<\theta_{i}<2 \pi, \Omega=D(0, a), r=a>0$ and $\Theta=\theta \in[0,2 \pi], t>0$ are defined as in (Fig. 5).

Let the eigenfunctions and eigenvalues concerning the region $\Gamma=D\left(a, \theta_{i}\right)_{2 \leq i \leq q}$ of $\partial \Omega$ with $\theta_{i} \in[0,2 \pi]$ are defined by

$$
\lambda_{n m}=-\beta_{n m}^{2}, n \geq 0, m \geq 1
$$

where $\beta_{n m}$ are the zeros of the Bessel functions $J_{n}$ and 


$$
\begin{array}{ll}
\varphi_{n m}(r, \theta)=J_{0}\left(\beta_{n m}^{2} r\right) & m \geq 1 \\
\varphi_{n m_{1}}(r, \theta)=J_{n}\left(\beta_{n m_{1}}^{2} r\right) \cos (n \theta) & n, m_{1} \geq 1 \\
\varphi_{n m_{2}}(r, \theta)=J_{n}\left(\beta_{n m_{2}}^{2} r\right) \sin (n \theta) & n, m_{2} \geq 1
\end{array}
$$
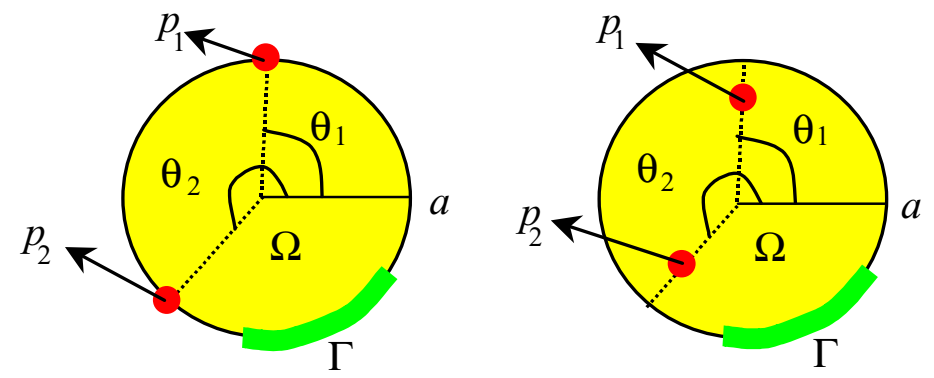

Figure 5. Domain $\Omega$, region $\Gamma$ and locations $p_{1}, p_{2}$ of internal (boundary) zone sensors.

with multiplicity $s_{n m}=2$ for all $n m \neq 0$ and $s_{n m}=1$ for all $n m=0$. In this case, the $\Gamma$-strategic sensor is required at least two zone sensors $\left(D_{i}, f_{i}\right)_{2 \leq i \leq q}$ where $D_{i}=\left(r_{i}, \theta_{i}\right)_{2 \leq i \leq q}$ (see [6]). The dynamical system (5.4) can be written by

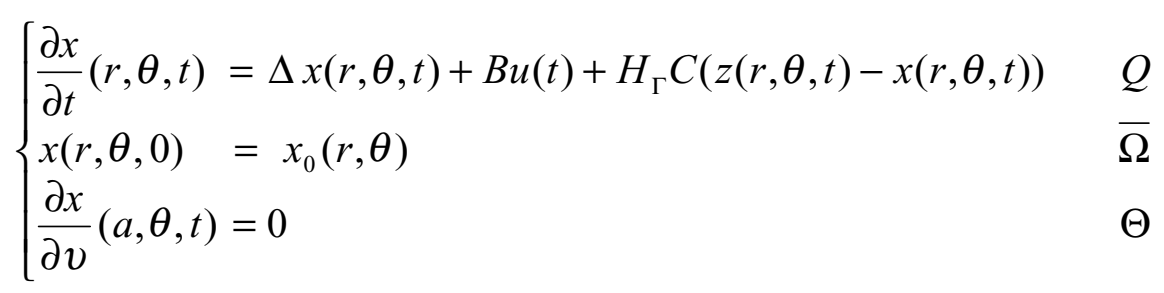

If $f_{i}$ and $D_{i}$ are symmetric with respect to $\theta=\theta_{i}$, for all $2 \leq i \leq q$, then we have :

Corollary 5.3. The dynamical system (5.8) is $\Gamma$-observer for the system (5.5) if $n\left(\theta_{1}-\theta_{2}\right) / \pi \notin N$ for every $n, n=1, \ldots, J$.

When the sensors $\left(\Gamma_{i}, f_{i}\right)_{2 \leq i \leq q}$ are located on $\partial \Omega$ and the function $f_{\left.\right|_{\Gamma_{i}}}$ is symmetric with respect to $\theta=\theta_{i}, 2 \leq i \leq q$ as in (Fig. 5). So, we have.

Corollary 5.4. The dynamical system (5.8) is $\Gamma$ - observer for the system (5.5) if $n\left(\theta_{1}-\theta_{2}\right) / \pi \notin N$ for every $n, n=1, \ldots, J$.

\subsection{Case a pointwise sensor}

In this subsection, we consider the following cases : 
5.2.1 The domain $\Omega=] 0, a_{1}[\times] 0, a_{2}[$

Now the system (5.5) is given by the form

$\left\{\begin{array}{lll}\frac{\partial z}{\partial t}\left(\xi_{1}, \xi_{2}, t\right) & =\Delta z\left(\xi_{1}, \xi_{2}, t\right)+B u(t) & Q \\ z\left(\xi_{1}, \xi_{2}, 0\right) & =z_{0}\left(\xi_{1}, \xi_{2}\right) & \bar{\Omega} \\ \frac{\partial z}{\partial v}\left(\eta_{1}, \eta_{2}, t\right) & =0 & \Theta \\ y(t) & =\int_{\Omega} z\left(\eta_{1}, \eta_{2}\right) f\left(\eta_{1}, \eta_{2}\right) d \eta_{1} d \eta_{2} & \Sigma\end{array}\right.$

If $b=\left(b_{1}, b_{2}\right) \in \partial \Omega$ then, we have :
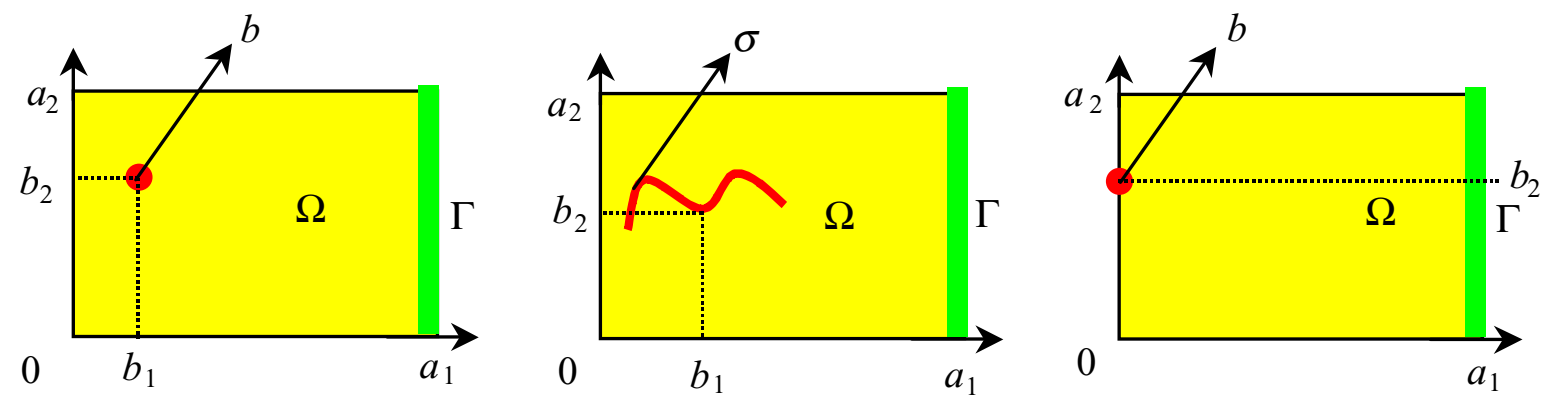

Figure 6. Rectangular domain, region $\Gamma$ and locations $b, \sigma$ of pointwise sensors.

\section{Corollary 5.5.}

1. Internal case : If $n b_{1} / a_{1}$ and $m b_{2} / a_{2} \notin N$ for every $n, m=1, \ldots, J$, then the dynamical system (5.4) is $\Gamma$-observer for the system (5.9).

2. Filament case : Suppose that the observation is given by the filament sensor where $\sigma=\operatorname{Im}(\gamma)$ is symmetric with respect to the line $b=\left(b_{1}, b_{2}\right)$, if $n b_{1} / a_{1}$ and $m b_{2} / a_{2} \notin N$ for every $n, m=1, \ldots, J$, the dynamical system (5.4) is $\Gamma$-observer for the system (5.9).

3. Boundary case : If $m b_{2} / a_{2} \notin N$ for every $m=1, \ldots, J$, then the dynamical system (5.4) is $\Gamma$ observer for the system (5.9).

\subsubsection{The domain $\Omega=\mathrm{D}(0, a)$}

The system (5.5) may be given by the following form

$$
\left\{\begin{array}{lll}
\frac{\partial z}{\partial t}(r, \theta, t) & =\Delta z(r, \theta, t)+B u(t) & \\
z(r, \theta, 0) & =z_{0}(r, \theta) & \bar{\Omega} \\
\frac{\partial z}{\partial v}(a, \theta, 0) & =0 & \Theta \\
y_{i}(t) & =\int_{\Omega} z(r, \theta, t) \delta_{p_{i}}\left(r-r_{i}, \theta-\theta_{i}\right) d r_{i} d \theta_{i} & \Sigma
\end{array}\right.
$$


where $0 \leq \theta_{i} \leq 2 \pi$. The sensors may be located in $p_{1}=\left(r_{1}, \theta_{1}\right)$ and $p_{2}=\left(r_{2}, \theta_{2}\right) \in \Omega$ (or in $\left.p_{i}=\left(a, \theta_{i}\right) \in \Omega\right)($ Fig. 7$)$.
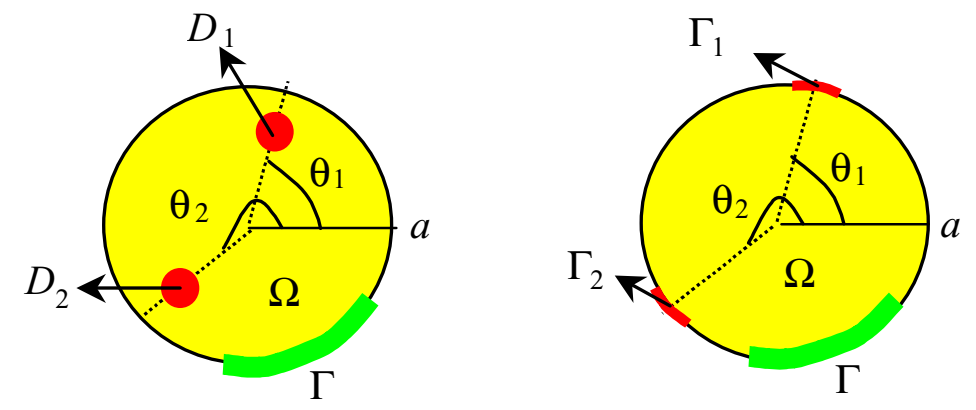

Figure 7. Disc domain, region $\Gamma$ and locations $p_{1}, p_{2}$ of internal (boundary) pointwise sensors.

\section{Corollary 5.6.}

1. If the sensors $\left(p_{i}, \delta_{p_{i}}\right)_{2 \leq i \leq q}$ are located in $p_{i}=\left(r_{i}, \theta_{i}\right)$ and $n\left(\theta_{1}-\theta_{2}\right) / \pi \notin N$ for every $n, n=1, \ldots, J$, then the dynamical system (5.8) is $\Gamma$-observer for the system (5.10).

2. If the sensors $\left(p_{i}, \delta_{p_{i}}\right)$ are located in $p_{i}=\left(a, \theta_{i}\right)_{2 \leq i \leq q}$ and $n\left(\theta_{1}-\theta_{2}\right) / \pi \notin N$ for every $n, n=1, \ldots, J$, then the dynamical system (5.8) is $\Gamma$-observer for the system (5.10).

\section{Conclusion}

The concept studied in this paper is related to the case of identity $\Gamma$-observer in connection with the sensors structure. For this class of parabolic distributed parameter systems, many interesting results concerning the choice of the sensor are explored and illustrated in specific situations of the domain. In similar way, we can characterize the general case (reduced-order) $\Gamma$-observer by sensors structure. An important extension of these results, related to the problem of regional boundary gradient observer, in connection with the structure of sensors; is under consideration.

\section{References}

1. Luenberger, D. Observers for multi-variable systems. IEEE Transactions on Automatics Control 1966, 11, 190-197.

2. Gressang, R.; Lamont, G. B. Observers for systems characterized by semi-groups. IEEE Transactions on Automatics Control 1975, 20, 523-528.

3. Fujii, N.; Hirai, M. A finite-dimensional asymptotic observer for a class of distributed parameter systems. International Journal of Control 1980, 32, 951-961.

4. Hou, M.; Muller, P.C. Design of observers for linear systems with unknown inputs, IEEE Transactions Automatic Control 1992, 37, 871-875.

5. Curtain, R. F.; Zwart, H. J. Introduction to infinite dimensional linear theory systems; SpringerVerlag : New York, 1995. 
6. El Jai, A.; Pritchard, A. J. Sensors and actuators in distributed parameter systems International Journal of Control 1987, 46, 1139-1153.

7. El Jai, A.; Pritchard, A. J. Sensors and controls in the analysis of distributed systems; Ellis Horwood series in Mathematics and its Applications, Wiley : New York, 1988.

8. El Jai, A.; Simon, M. C.; Zerrik, E. Regional observability and sensor structures. Sensors and Actuators 1993, 39, 95-102.

9. Zerrik, E.; Badraoui, L.; El Jai, A. Sensors and regional boundary state reconstruction of parabolic systems. Sensors and Actuators 1999, 75, 102-117.

10. El Jai, A.; Zerrik, E.; Simon, M. C.; Amouroux, M. Regional observability of a thermal process. IEEE Transactions on Automatic Control 1995, 40, 518-521.

11. Al-Saphory, R.; El Jai, A. Sensors and asymptotic $\omega$-observer for distributed diffusion systems. Sensors 2001, 1, 161-182.

12. Al-Saphory, R.; El Jai, A. Sensors structures and regional detectability of parabolic distributed systems. Sensors and Actuators 2001, 29, 163-171.

13. Al-Saphory, R.; El Jai, A. Sensors characterizations for regional boundary detectability of distributed parameter systems. Sensors and Actuators 2001, 94, 1-10.

14. Al-Saphory, R.; El Jai, A. Asymptotic regional state reconstruction. International Journal of Systems Science 2002 to appear.

15. Curtain, R. F. Finite dimensional compensators for parabolic distributed systems with unbounded control and observation. SIAM J. Control and Optimisation 1984, 22, 255-275.

16. Zerrik, E.; Badraoui, L. Sensors characterization for regional boundary observability. International Journal of Applied Mathematics and Computer Science 2000, 10, 345-356.

17. Zerrik, E.; Boutoulout, L.; El Jai, A. Actuators and regional boundary controllability of parabolic systems. International Journal of Systems Science 2000, 31, 73-82.

18. Al-Saphory, R. Asymptotic regional analysis for a class of distributed parameter systemes, Ph.D. thesis, University of Perpignan, France, 2001.

19. Dautray R.; Lions, J.L. Analyse mathématique et calcul numérique pour les sciences et les techniques; série scientifique 8, Masson : Paris, 1984.

20. Kitamura, S.; Sakairi, S.; Nishimura, M. Observer for distributed-parameter diffusion systems. Electrical engineering in Japan 1972, 92, 142-149.

21. El Jai, A.; El Yacoubi, S. On the number of actuators in parabolic system, International Journal of Applied Mathematics and Computer Science 1993, 3, 673-686.

Sample Availability: Available from the authors.

(C) 2002 by MDPI (http://www.mdpi.net). Reproduction is permitted for noncommercial purposes. 